\title{
The nucleotides that bind
}

\author{
Rapid progress is being made in our understanding of RNA-protein interactions, their role in disease and their \\ influence on the action of oligonucleotide drugs.
}

I he recent virtual Nature Conference 'RNA at the Bench and Bedside II' provided a fascinating snapshot of research aimed at deciphering RNA-protein interactions in cells. An increasingly powerful set of analytical tools now offers the chance to catalog RNAs and RNA-binding proteins (RBPs) on a transcriptome-wide scale. These analyses are revealing the myriad ways in which RBPs interact with RNAs, organize into subcellular condensates, drive biological processes and ultimately contribute to disease. This is not only informing our understanding of basic aspects of the RNA life cycle (splicing and processing, transport, localization, modification and degradation) but also revealing what happens when antisense oligonucleotides (ASOs) enter cells, why certain ASO molecules are toxic and, critically, how medicinal chemistry can be used to circumvent toxicity. This is of relevance to any developer of an RNA therapy with a similar phosphorothioate chemistry.

RNA at the Bench and Bedside II was organized by Nature Biotechnology and Nature Structural \& Molecular Biology in partnership with the University of California, San Diego and Ionis Pharmaceuticals. The conference ran for three days and attracted over 500 registrants. The program mixed talks from researchers working on basic aspects of RNA biology, drug makers developing therapies to target RNAs, and clinicians testing RNA therapies in humans.

An impressive array of techniques was on display, illustrating that RNA-RBP analysis in cells is now possible in vivo at a throughput and scale unimaginable just a few years ago. A raft of methods (for example, PARS, DMS-seq, RNA proximity ligation, PARIS, LIGR-seq and SHAPE) combine chemical probes with deep sequencing to analyze RNA secondary structure and RBP interactions. In addition, ultraviolet crosslinking of RBPs to RNAs can be tracked either on a proteome-wide level via mass spectrometry (oligo(dT), RICK or CARIC, RBR-ID and TRAPP) or on a transcriptome-wide level via high-throughput sequencing readout (HITS-CLIP, CRAC, iCLIP, PAR-CLIP and eCLIP). Going forward, single-cell methods promise to allow analysis of ribonucleoprotein (RNP) complexes in specific tissues and cell types.

Two recent publications (in Nature and Genome Biology) from the ENCODE 3 project provide eCLIP data on 365 RBPs the most comprehensive dataset collected, albeit still just scratching the surface of the $>1,500$ RBPs in human cells. These data are starting to provide a broader picture of what RBP-RNA interactions look like collectively.

From this new perspective, RBPs display a broad range of regional specifities across the length of an RNA molecule; different members of the same RBP family are found to bind RNAs in similar places; and the location of the binding site - whether within, upstream of or downstream of a coding region, for example - can provide clues as to RBP function. Certainly, the list of canonical consensus motifs (for example, RNA recognition motif (RRM), zinc fingers or DEAD-box helicase domain) found in RBPs continues to grow, but it is becoming increasingly clear that RNA-binding domains alone are not the whole story; indeed, 55\% of RBPs do not contain any known RNA-binding domain at all.

Overall, multiple RBP consensus motifs with RNA secondary/tertiary structure and RNA chemical modifications all can contribute to RNA-protein interactions, with several proteins often working in concert; indeed, many RBPs show specific low-affinity binding for RNAs due to intrinsically disordered regions (IDRs). In turn, these IDRs are thought to drive formation of membraneless structures in cells undergoing liquid-liquid phase separation.

This brings us to the meeting's second major theme: how RNP complexes localize in cells - whether in the form of active macromolecular complexes (for example, the spliceosome or ribosome) or in RNP granules and condensates.

After nuclear processing, mRNAs are exported to the cytoplasm and either translated, degraded, stored in P-bodies or transported to a site of localized translation (in, for example, neurons or the developing embryo). Cellular stress stalls translation, disengages ribosomes and sequesters RNA-protein translation complexes in stress granules. In some neurodegenerative disorders, the propensity for condensate formation increases, with mutations in RNP granule components, such as TDP-43 and FUS, driving deposition. Until recently, this was thought to be primarily a protein-driven process. But it turns out RNA aggregation might be just as important in diseases like Alzheimer's, where tau aggregates contain high levels of small nuclear RNAs and small nucleolar RNAs. The redistribution of RBPs into cytoplasmic condensates is thought to deplete levels in the nucleus, leading to aberrant mRNA splicing or polyadenylation. One intriguing study suggests that the RBP helicase eIF4A may have the capacity to reverse RNA aggregation (analogously to how HSP70 refolds proteins). In addition, ASOs have been shown to reverse neurotoxic TDP-43 assemblies associated with amyotrophic lateral sclerosis and frontotemporal dementia both in vitro and in vivo.

This leads to a final thought from the conference. While new analytical tools are improving our understanding of RBPRNA interactions in cells, the systematic analysis of cellular protein binding to phosphorothioate ASOs has provided new insights into how these drugs are internalized, released from endosomes and trafficked through cells - information that is now being exploited to improve pharmacodynamics and pharmacokinetics. Protein aggregation has also been identified as one of the most important mechanisms of ASO toxicity. By aggregating proteins, such as p54nrb and PSF, toxic ASOs accelerate the accumulation of damaging condensates in the nucleolus.

Drug developers should take note. These findings are not just of esoteric interest to ASO drug programs. Every RNA therapeutic currently under development - whether a small-interfering RNA, an RNA-guided CRISPR endonuclease or deaminase, a modified mRNA or an antagomiR employs phosphorothioate chemistry in some part of the molecule. We need to know which intracellular proteins nucleic acid therapeutics are binding and where in cells the complexes are ending up.

Published online: 12 January 2021 https://doi.org/10.1038/s41594-020-00552-8 